\title{
Editorial
}

\section{Advances in the Diagnosis and Management of Oral Diseases}

\section{INTRODUCTION}

The supplement is intended to provide practicing dentists, dental educators, and students with an overview of recent advances in the diagnosis and management of oral diseases. The first three articles cover advances in the diagnostic approaches for oral cancer, candidiasis, and contact allergic mucositis of the mouth. Articles four through six focus on treatment approaches for oral mucosal diseases, advances in ridge augmentation, and oral and maxillofacial reconstruction prostheses. Below is a brief description of topics covered in the supplement:

\section{Advances in the Diagnostic Adjunct for Oral Squamous Cell Carcinoma}

Oral cancer is a common malignancy; current statistics indicates that approximately $24 \%$ of the 22,000 Americans affected by oral cancer die prematurely because of its complications. Despite the advances in the diagnosis and treatment of malignant lesions, the five-year survival rate for oral cancer remains the lowest among all of the major cancers (53-60\%). The delayed diagnosis of the cancerous lesions in the mouth is thought to be one of the major reasons for the low survival rate. Clinicians and researchers continue striving to improve early diagnosis of oral malignant lesions. Currently available diagnostic tools for oral cancer include tissue staining with toludine blue or tulonium chloride, brush cytology and tissue autofluorescence. New research frontier suggests that salivary-based diagnostics show considerable promise for early detection of oral cancer. Microfluidics and Nanosensor are among the recently developed devices that are being investigated as salivary-based diagnostics for oral cancer. Drs. Cheng and Wright report on recent advances in the diagnostic adjuncts for early detection of oral malignant lesions.

\section{Hypersensitivity to Cast Metal Dental Restorations: A Potential Etiologic Factor in Mucosal and Periodontal Diseases}

Intraoral contact allergy to food and drinks; oral hygiene products; and dental materials and metals, is a fairly common problem in dentistry. Contact allergy is believed to play an integral role in the development of lichen planus and lichenoid lesions of the mouth. There is generally a temporal relationship between the development of oral symptoms and the exposure to the allergen. However, this relationship can potentially be modified by the potency of the allergen, and local factors such as the thickness of the keratinized mucosa, salivary flow, trauma, and type of food and drinks. A through history and careful clinical examination are key for the diagnosis. Skin patch test is not always helpful for the diagnosis of contact allergy of the mouth because, depending on the type of the allergen, the reaction may remain localized in the mouth for a long time before it is manifested on the skin. Dr. Rees reports on 442 dental patients with hypersensitive to cast dental restorations. Fifty percent of the patients with hypersensitivity developed hyperplastic gingivitis and periodontitis.

\section{Advances and Emerging Techniques for the Diagnosis and Treatment of Oral Candidiasis}

Candidiasis is a common oral infection; most frequently associated with Candida albicans, which occurs in both pathogenic and nonpathogenic forms within the oral cavity. Candida species have developed powerful mechanisms for colonization of the mouth under permissible conditions. Recent advances in cell-molecular biology have helped identifying genes that regulate the communication between epithelial cells involved in oropharyngeal candidiasis and vascular endothelial cells. Employing polymerase chain reaction and recombinant DNA techniques have enhanced our understanding of the mechanisms involved in the endurance and continued existence of oral candidiasis. Dr. Abraham's review covers various cellular and molecular mechanisms that might be involved in the interactions between Candida organisms with their environment, development of oral candidal infections, and current treatment modalities for oral candidiasis.

\section{Treatment Options for Common Mucocutaneous Lesions}

Reactive lesions of the gingiva are common and have a relatively high incidence of recurrence after their removal. Treatment of reactive lesions of the gingiva includes surgical excision, laser therapy, and electrosurgery. Dr. Rossmann reviews selected cases of reactive gingival lesions and methodologically demonstrates development of diagnosis, treatment plan, and surgical approaches to ensure successful treatment outcomes.

\section{Advances in Predictable Guided Bone Regeneration}

Guided bone regeneration, typically refers to ridge augmentation and bone regeneration, was evolved from guided tissue regeneration techniques that are directed toward regenerating tissues in osseous defects adjacent to natural teeth. These techniques are used to stimulate new bone formation and to treat defects associated with teeth extractions. Advances in guided bone regeneration has allowed for placement of restorations at a more ideal locations in the oral cavity thereby improving esthetics and functions. Drs. Hitti and Kerns review current techniques used for ridge augmentation and preservation. 


\section{The Impact of Dental Implants on Patients' Quality of Life}

Surgery for benign and malignant lesions often leaves defects in the jaw and facial structures. Such defects can compromise patients' quality of life and wellbeing because of facial deformities and interference with oral functions such as eating and talking. Advances in maxillofacial implants have been successful in restoring esthetics and functional problems. Dr. Gonzales presents cases to demonstrate current approaches used for maxillofacial restorations along with a brief review of technological advances in bone grafting and tissue engineering of the bone.

Finally, the authors of the reviews are educators and practicing dentists, it is our hope that our colleagues and students find this supplement informative and useful.

Ibtisam Al-Hashimi

(Guest Editor)

Salivary Dysfunction Clinic

Department of Periodontics

Baylor College of Dentistry

3302 Gaston Avenue

Dallas

TX 75246

USA

E-mails: IAl-Hashimi@bcd.tamhsc.edu

(C) Ibtisam Al-Hashimi; Licensee Bentham Open.

This is an open access article licensed under the terms of the Creative Commons Attribution Non-Commercial License (http: //creativecommons.org/licenses/by-nc/ 3.0/) which permits unrestricted, non-commercial use, distribution and reproduction in any medium, provided the work is properly cited. 